\title{
High Yield Solution-Liquid-Solid Synthesis of Germanium Nanowires
}

\author{
Xianmao Lu, Dayne D. Fanfair, Keith P. Johnston*, Brian A. Korgel* \\ Department of Chemical Engineering, Texas Materials Institute, Center for Nano- and Molecular \\ Science and Technology, The University of Texas at Austin, Austin, Texas 78712-1062
}

\section{Supporting Information.}

Ge nanowire synthesis. Ge nanowires were synthesized under nitrogen in a three-neck flask on a Schlenk line. GeI $_{2}$ (Strem Chemicals, packed under argon) was stored in a nitrogenfilled glovebox upon purchase. Tri-n-octylphosphine (Tech. grade, Aldrich) was degassed through a freeze-pump-thaw technique and distilled at $200^{\circ} \mathrm{C}$ and 0.3 Torr. All other solvents were used as received from Fisher Scientific without further purification. A precursor solution of $0.3 \mathrm{M} \mathrm{GeI}_{2}$ in TOP was prepared by adding $0.2 \mathrm{~g} \mathrm{GeI}_{2}$ into $2 \mathrm{ml}$ TOP in the glovebox. A clear yellow solution was formed after stirring for $1 \mathrm{hr}$. Bi nanocrystals were prepared following the procedures described by Fanfair and Korgel. ${ }^{1} \quad 0.6 \mathrm{mg}$ Bi nanoparticles were mixed with $0.5 \mathrm{ml}$ TOP and sonicated for $2 \mathrm{hrs}$ to form a grey dispersion. $0.5 \mathrm{ml} \mathrm{Bi}$ nanoparticle dispersion and desired volume of $\mathrm{GeI}_{2}$ solution in TOP $\left(0.8 \mathrm{ml}\right.$ for $\mathrm{GeI}_{2}$ to Bi mole ratio of $80: 1,0.5 \mathrm{ml}$ for a ratio of 50:1) were loaded into a $3 \mathrm{ml}$ plastic syringe. $4 \mathrm{ml}$ TOP was loaded into a 3-neck flask and heated to $365^{\circ} \mathrm{C}$ under $\mathrm{N}_{2}$, followed by the injection of the $\mathrm{GeI}_{2}$ and $\mathrm{Bi}$ nanocrystal solution through a rubber septum with stirring. The introduction of the precursor and Bi nanocrystals immediately produced a gray solution. The temperature dropped to $350^{\circ} \mathrm{C}$ and the reaction was allowed to proceed at $350^{\circ} \mathrm{C}$ for 10 min before taking the reaction flask off the heating mantle and allowing it to cool to room temperature. The nanowire dispersion was diluted with $10 \mathrm{~mL}$ toluene and centrifuged at $8,000 \mathrm{rpm}$ for $5 \mathrm{~min}$. The nanowires in the black precipitate were redispersed in $2.5 \mathrm{ml} \mathrm{CHCl}_{3}$ and precipitated with $2.5 \mathrm{ml}$ ethanol and centrifuged again to obtain 
a purified nanowire product. Nanowires were dried on a rotary evaporator and stored under nitrogen prior to characterization.

Materials Characterization. The nanowires were characterized by scanning electron microscopy (SEM), transmission electron microscopy (TEM), energy-dispersive X-ray spectroscopy (EDS) and powder X-ray diffraction (XRD). SEM samples were prepared by drop casting chloroform-dispersed nanowires onto Si substrates. SEM was performed on a LEO 1530 field emission gun SEM, operating at $10 \mathrm{kV}$ accelerating voltage, and digital SEM images were acquired using an inlens detector and LEO 32 software system. TEM specimens were prepared by drop casting dilute dispersions of nanowires after washing with $5 \% \mathrm{HCl}$ acid for 5 min onto 200-mesh copper grids coated with lacy carbon film (Ladd Research Co.). High resolution TEM (HRTEM) was performed on a JEOL 2010F TEM; images were obtained digitally with a GATAN digital photography system. EDS was performed on the JEOL 2010F HRTEM equipped with an Oxford spectrometer. X-ray diffraction (XRD) was obtained from $\sim 2 \mathrm{mg}$ nanowires deposited by drop casting on quartz slides (Gem Dugout, State College, PA) with continuous scan for 3 hours at a rate of $12^{\circ} / \mathrm{min}$ and $0.02^{\circ}$ per step using a Bruker-Nonius D8 Advance powder diffractometer with $\mathrm{Cu} K_{\alpha}$ radiation $(\lambda=1.54 \AA)$ and a rotary sample stage (30rpm). 


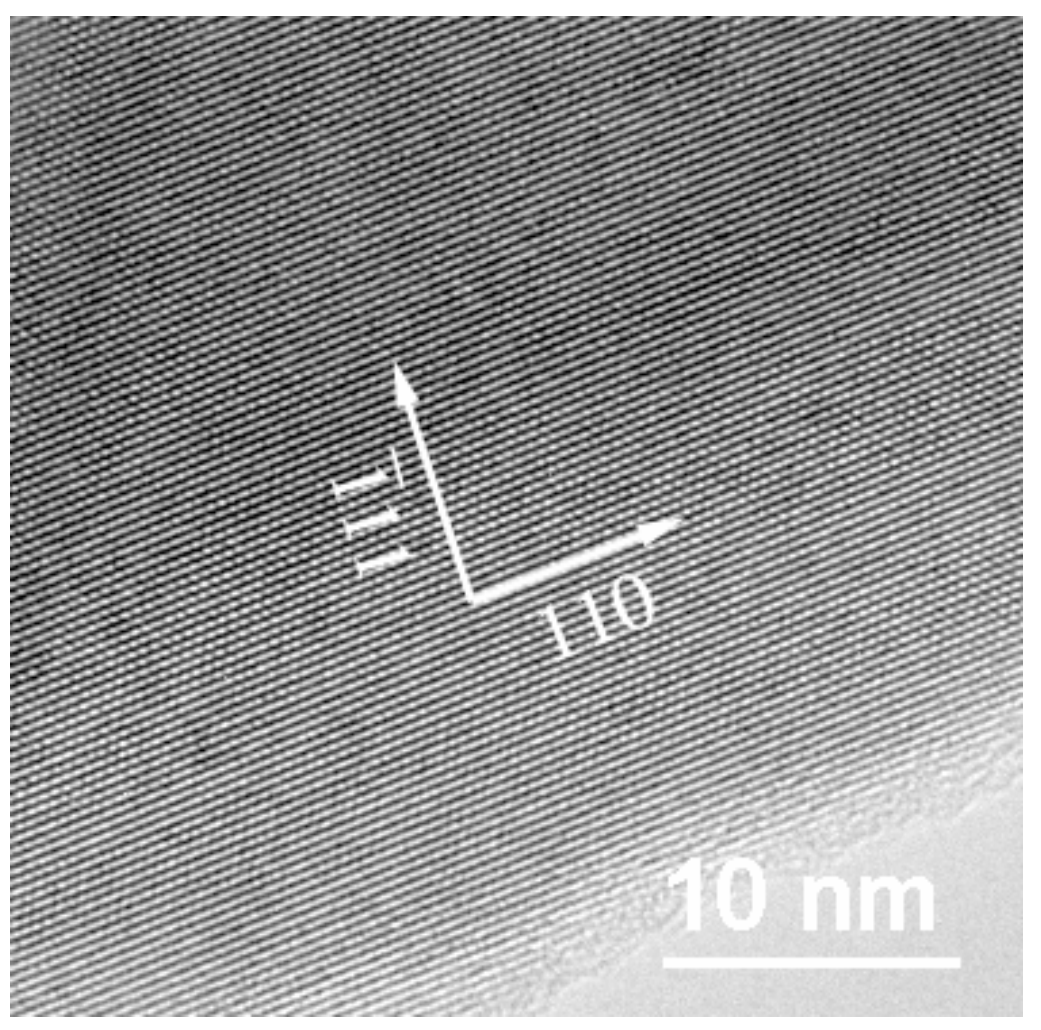

FigureSI-1. HRTEM image of a Ge nanowire showing a $<110>$ growth direction. 

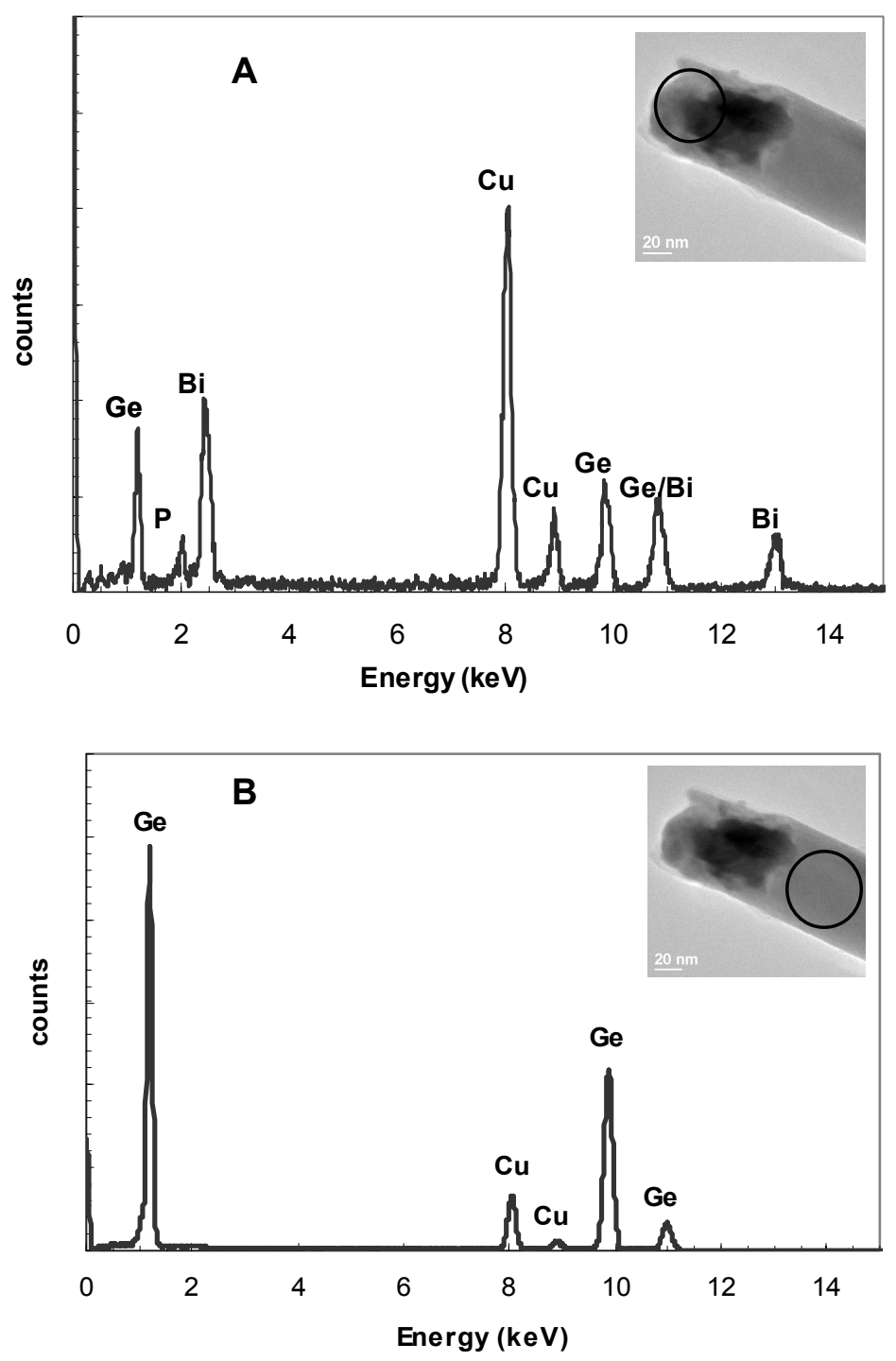

Figure SI-2. EDS of a Ge nanowire: the black circles drawn in the TEM images in the insets indicate the beam position. (A) EDS of the nanowire tip shows both $\mathrm{Ge}$ and $\mathrm{Bi}$ with a ratio of $\sim 1: 1$ (Ge:Bi). The peaks of $\mathrm{Cu}$ and $\mathrm{P}$ are from the copper TEM grid and the solvent of TOP used in the reaction, respectively. (B) EDS taken from the nanowire core shows only Ge.

\section{References}

1. Fanfair, D. D.; Korgel, B. A., Crystal Growth \& Design 2005, 5, 1971. 